\title{
Hochschild (co)homology of exterior algebras ${ }^{1}$
}

\author{
Yunge $\mathrm{Xu}{ }^{a}$, Yang $\operatorname{Han}^{b, 2}$ and Wenfeng Jiang ${ }^{a}$ \\ a. Faculty of Mathematics \& Computer Science, Hubei University, \\ Wuhan 430062, P.R.China E-mail: xuy@hubu.edu.cn \\ b. Academy of Mathematics and Systems science, Chinese Academy of Sciences, \\ Beijing 100080, P.R. China E-mail: hany@iss.ac.cn \\ Dedicated to Professor Yingbo Zhang on the occasion of her 60th birthday
}

\begin{abstract}
The minimal projective bimodule resolutions of the exterior algebras are explicitly constructed. They are applied to calculate the Hochschild (co)homology of the exterior algebras. Thus the cyclic homology of the exterior algebras can be calculated in case the underlying field is of characteristic zero. Moreover, the Hochschild cohomology rings of the exterior algebras are determined by generators and relations.
\end{abstract}

Keyword: Exterior algebra, Hochschild (co)homology, minimal projective resolution

MSC(2000): 16E40, 16G10

\section{Introduction}

Fix a field $k$. Let $\Lambda$ be a finite-dimensional $k$-algebra (associative with identity). Denote by $\Lambda^{e}$ the enveloping algebra of $\Lambda$, i.e., the tensor product $\Lambda \otimes_{k} \Lambda^{o p}$ of the algebra $\Lambda$ and its opposite $\Lambda^{o p}$. The Hochschild homology and cohomology of $\Lambda$ are defined by

$$
H H_{m}(\Lambda)=\operatorname{Tor}_{m}^{\Lambda^{e}}(\Lambda, \Lambda) \text { and } H H^{m}(\Lambda)=\operatorname{Ext}_{\Lambda^{e}}^{m}(\Lambda, \Lambda)
$$

respectively 22]. The Hochschild (co)homology of an algebra have played a fundamental role in representation theory of artin algebras: Hochschild cohomology is closely related to simple connectedness, separability and deformation theory [26, 1, 12] Hochschild homology is closely related to the oriented cycle and the global dimension of algebras [17, 19, 3, 14, 18.

So far the Hochschild cohomology was calculated for hereditary algebras [16, incidence algebras [7, 13], algebras with narrow quivers [16, 8], radical

\footnotetext{
${ }^{1}$ Project 10426014 and 10201004 supported by NSFC

${ }^{2}$ Corresponding author
} 
square zero algebras [9, monomial algebras [10], truncated quiver algebras [11, 29, 20, and special biserial algebras as well as their trivial extensions [27, 15]. The Hochschild homology was calculated for truncated algebras 19, 30, 25, quasi-hereditary algebras 28 and monomial algebras 14 .

The exterior algebras play extremely important roles in many mathematical branches such as algebraic geometry, commutative algebra, differential geometry. However, up to now their Hochschild (co)homology are still unknown. In this paper we shall deal with this problem. In section 1, we shall provide the minimal projective bimodule resolutions of the exterior algebras. In section 2, we shall apply these minimal projective bimodule resolutions to calculate the Hochschild homology of the exterior algebras and their cyclic homology in case the underlying field is of characteristic zero. In section 3, we shall apply these minimal projective bimodule resolutions to calculate the Hochschild cohomology of the exterior algebras. In section 4, we shall determine the Hochschild cohomology rings of the exterior algebras by generators and relations.

\section{Minimal projective bimodule resolutions}

Let $Q$ be the quiver given by one point 1 and $n$-loops $x_{1}, x_{2}, \ldots, x_{n}$ with $n \geq 2$. Denote by $I$ the ideal of the path algebra $k Q$ generated by $R:=\left\{x_{i}^{2} \mid 1 \leq i \leq\right.$ $n\} \cup\left\{x_{i} x_{j}+x_{j} x_{i} \mid 1 \leq i<j \leq n\right\}$. For the knowledge on quiver we refer to [2]. Set $\Lambda=k Q / I$. Then $\Lambda$ is just the exterior algebra over $k$ (cf. [23]). Order the paths in $Q$ by left length lexicographic order by choosing $1<x_{1}<x_{2}<$ $\cdots<x_{n}$, namely, $y_{1} \cdots y_{s}<z_{1} \cdots z_{t}$ with $y_{i}$ and $z_{i}$ being arrows if $s<t$ or if $s=t$, for some $1 \leq r \leq s, y_{i}=z_{i}$ for $1 \leq i<r$ and $y_{r}<z_{r}$. Then $\Lambda$ has a basis $\mathcal{B}=\cup_{i=0}^{n} \mathcal{B}_{i}$, where $\mathcal{B}_{i}=\left\{x_{t_{1}} x_{t_{2}} \cdots x_{t_{i}} \mid 1 \leq t_{1}<t_{2}<\cdots<t_{i} \leq n\right\}$. So $\operatorname{dim}_{k} \Lambda=2^{n}$. It is well-known that $\Lambda$ is a Koszul algebra and its quadratic duality is just the algebra of polynomials $k\left[x_{1}, \ldots, x_{n}\right]$ (cf. [4]).

Now we construct a minimal projective bimodule resolution $\left(P_{\bullet}, \delta_{\bullet}\right)$ of $\Lambda$. Denote by $k\left\langle x_{1}, \ldots, x_{n}\right\rangle$ the noncommutative free associative algebra over $k$ with free generators $x_{1}, \ldots, x_{n}$. Denote by $k\left\langle x_{1}, \ldots, x_{n}\right\rangle_{m}$ the $k$-subspace of $k\left\langle x_{1}, \ldots, x_{n}\right\rangle$ generated by all monomials of degree $m$. For each $m \geq 0$,

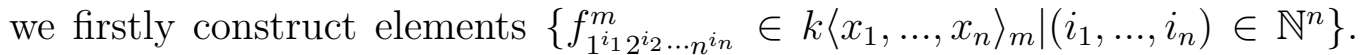
Let $f_{0}^{0}=1, f_{1}^{1}=x_{1}, f_{2}^{1}=x_{2}, \ldots, f_{n}^{1}=x_{n}$. Define $f_{1^{i_{1}} 2^{i_{2} \ldots n{ }^{n}}}^{m}$ for all $m \geq 2$

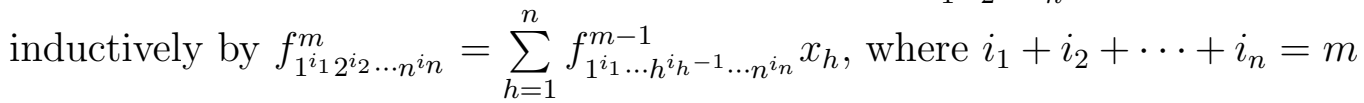
with $i_{h} \geq 0$ and $f_{1^{i_{1} \ldots h^{-1} \ldots n^{i n}}}^{m-1}=0$ for all $1 \leq h \leq n$. It is well-known that the number of non-negative integral solutions of the equation $i_{1}+i_{2}+\cdots+i_{n}=m$ on $i_{1}, \ldots, i_{n}$ is $\left(\begin{array}{c}n+m-1 \\ n-1\end{array}\right)$ for any given positive integers $n$ and $m$. 
Denote $\otimes:=\otimes_{k}$. Let $P_{m}:=\coprod_{i_{1}+i_{2}+\cdots+i_{n}=m} \Lambda \otimes f_{1^{i_{1}} 2^{i_{2} \cdots n^{i n}}} \otimes \Lambda \subseteq \Lambda \otimes$ $k\left\langle x_{1}, \ldots, x_{n}\right\rangle_{m} \otimes \Lambda$ for $m \geq 0$, and let $\widetilde{f}_{1^{i_{1}} 2^{i_{2} \ldots n^{i n}}}^{m}:=1 \otimes f_{1^{i_{1}} 2^{i_{2} \ldots n^{i n}}}^{m} \otimes 1$ for $m \geq 1$ and $\widetilde{f}_{0}^{0}=1 \otimes 1$. Note that we identify $P_{0}$ with $\Lambda \otimes \Lambda$. Define $\delta_{m}: P_{m} \rightarrow P_{m-1}$ by setting

$$
\delta_{m}\left(\widetilde{f}_{1^{i_{1}} 2^{i_{2} \ldots n^{i n}}}\right)=\sum_{h=1}^{n}\left(x_{h} \widetilde{f}_{1^{i_{1} \ldots h^{i} h^{-1} \cdots n^{i n}}}^{m-1}+(-1)^{m} \widetilde{f}_{1^{i_{1} \ldots h^{i} h-1} \ldots n^{i n}}^{m-1} x_{h}\right) .
$$

Theorem 1. The complex $\left(P_{\bullet}, \delta_{\bullet}\right)$

$$
\cdots \rightarrow P_{m+1} \stackrel{\delta_{m+1}}{\longrightarrow} P_{m} \stackrel{\delta_{m}}{\longrightarrow} \cdots \stackrel{\delta_{3}}{\longrightarrow} P_{2} \stackrel{\delta_{2}}{\longrightarrow} P_{1} \stackrel{\delta_{1}}{\longrightarrow} P_{0} \longrightarrow 0
$$

is a minimal projective bimodule resolution of the exterior algebra $\Lambda=k Q / I$.

Proof. Now we consider the minimal projective bimodule resolution of $\Lambda$ constructed in [6, section 9]. Let $X=\left\{x_{1}, x_{2}, \cdots, x_{m}\right\}$. We show that

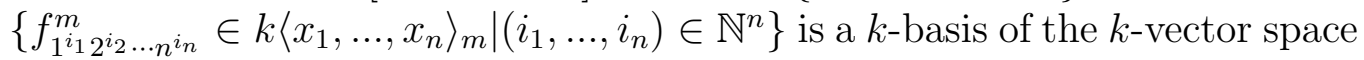
$K_{m}:=\bigcap_{p+q=m-2} X^{p} R X^{q}$.

Firstly, we verify $f_{1^{i_{1} 2^{i_{2} \ldots n^{i} n}}}^{m} \in K_{m}$ for all $m \geq 0$ : It is clear that $f_{1^{i_{1}} 2^{i_{2} \cdots n^{i_{n}}}}^{m}$ $=\sum_{h=1}^{n} f_{1^{i_{1} \ldots h^{i_{h}-1} \cdots n^{i_{n}}}}^{m-1} x_{h}=\sum_{h=1}^{n} x_{h} f_{1^{i_{1} \ldots h^{i_{h}-1} \cdots n_{n}}}^{m-1}$. Thus the assertion follows by induction on $m$.

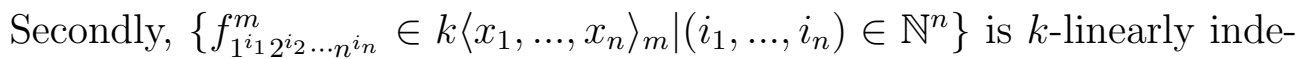
pendent.

Thirdly, we have $\operatorname{dim}_{k} K_{m}=\left(\begin{array}{c}n+m-1 \\ n-1\end{array}\right)$ : The quadratic duality of the Koszul algebra $\Lambda$ is just the algebra of polynomials $k\left[x_{1}, \ldots, x_{n}\right]$ which is isomorphic to the Yoneda algebra $E(\Lambda)=\coprod_{m \geq 0} \operatorname{Ext}_{\Lambda}^{m}(k, k)$ of $\Lambda$ (cf. [4, Theorem 2.10.1]). Thus $\operatorname{dim}_{k} K_{m}=\operatorname{dim}_{k} \operatorname{Ext}_{\Lambda}^{m}(k, k)=\left(\begin{array}{c}n+m-1 \\ n-1\end{array}\right)$. Hence $\left\{f_{1^{i_{1} 2^{i_{2} \cdots n^{i n}}}} \mid i_{1}+i_{2}+\cdots+\right.$ $\left.i_{n}=m\right\}$ is a $k$-basis of $K_{m}$.

Fourthly and finally, the maps $\delta$. is determined by [6, p.354].

\section{Hochschild homology}

In this section we calculate the $k$-dimension of Hochschild homology groups of the exterior algebras.

Applying the functor $\Lambda \otimes_{\Lambda^{e}}-$ to the minimal projective bimodule resolution $\left(P_{\bullet}, \delta_{\bullet}\right)$, we have $\Lambda \otimes_{\Lambda^{e}}\left(P_{\bullet}, \delta_{\bullet}\right)=\left(M_{\bullet}, \tau_{\bullet}\right)$ where $M_{m}=\coprod_{i_{1}+i_{2}+\cdots+i_{n}=m} \Lambda \otimes$ 


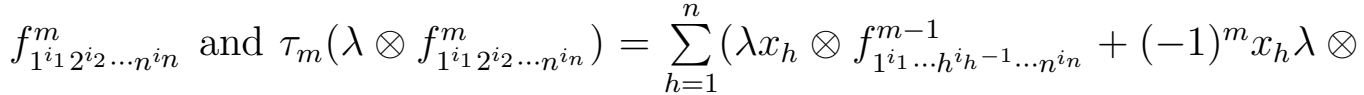

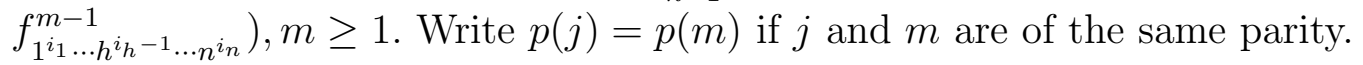

Lemma 1. For $m \geq 1$, we have

$$
\operatorname{rank} \tau_{m}= \begin{cases}\sum_{i=1}^{n}\left(\begin{array}{c}
n \\
i
\end{array}\right) \sum_{\substack{j=0 \\
p(j)=p(m)}}^{i-1}\left(\begin{array}{c}
j+m-1 \\
i-1
\end{array}\right)\left(\begin{array}{c}
i-1 \\
j
\end{array}\right), & \text { if char } k \neq 2 ; \\
0, & \text { otherwise. }\end{cases}
$$

Remark 1. Throughout we assume that the combinatorial number $\left(\begin{array}{l}0 \\ 0\end{array}\right)=$ 1 , and $\left(\begin{array}{l}i \\ j\end{array}\right)=0$ if $i<j$. Thus we have $\operatorname{rank} \tau_{m}=\sum_{i=1}^{n}\left(\begin{array}{c}n \\ i\end{array}\right) \sum_{\substack{j=0 \\ p(j)=p(m)}}^{i-1}\left(\begin{array}{c}j+m-1 \\ i-1\end{array}\right)\left(\begin{array}{c}i-1 \\ j\end{array}\right)$ if $m \geq n$ and char $k \neq 2 ; \quad \operatorname{rank} \tau_{m}=\sum_{i=1}^{m}\left(\begin{array}{c}n \\ i\end{array}\right) \sum_{\substack{j=0 \\ p(j)=p(m)}}^{i-1}\left(\begin{array}{c}j+m-1 \\ i-1\end{array}\right)\left(\begin{array}{c}i-1 \\ j\end{array}\right)+$ $\sum_{i=m+1}^{n}\left(\begin{array}{l}n \\ i\end{array}\right) \sum_{\substack{j=i-m \\ p(j)=p(m)}}^{i-1}\left(\begin{array}{c}j+m-1 \\ i-1\end{array}\right)\left(\begin{array}{c}i-1 \\ j\end{array}\right)$ if $1 \leq m<n$ and char $k \neq 2$; and $\operatorname{rank} \tau_{m}=0$ otherwise.

Proof. Denote by $k\left[x_{1}, \ldots, x_{n}\right]_{m}$ the subspace of $k\left[x_{1}, \ldots, x_{n}\right]$ generated by all monomials of degree $m$. Obviously the complex $\left(M_{\bullet}, \tau_{\bullet}\right)$ is isomorphic to the complex $\left(N_{\bullet}, \sigma_{\bullet}\right)$ which is defined by $N_{m}:=\Lambda \otimes k\left[x_{1}, \ldots, x_{n}\right]_{m}$ and $\sigma_{m}: N_{m} \rightarrow N_{m-1}, \lambda \otimes x_{1}^{i_{1}} \cdots x_{n}^{i_{n}} \mapsto \sum_{h=1}^{n}\left(\lambda x_{h} \otimes x_{1}^{i_{1}} \cdots x_{h}^{i_{h}-1} \cdots x_{n}^{i_{n}}+(-1)^{m} x_{h} \lambda \otimes\right.$ $\left.x_{1}^{i_{1}} \cdots x_{h}^{i_{h}-1} \cdots x_{n}^{i_{n}}\right)$.

For any $\lambda=x_{t_{1}} \cdots x_{t_{j}} \in \mathcal{B}$, define $\mu_{\lambda}(h):=(-1)^{\left|\left\{t_{l} \mid t_{l}<h, 1 \leq l \leq j\right\}\right|}$. Then $\sigma_{m}\left(\lambda \otimes x_{1}^{i_{1}} \cdots x_{n}^{i_{n}}\right)=\left((-1)^{j}+(-1)^{m}\right) \sum_{h=1}^{n}\left(\mu_{\lambda}(h) N\left(\lambda x_{h}\right) \otimes x_{1}^{i_{1}} \cdots x_{h}^{i_{h}-1} \cdots x_{n}^{i_{n}}\right)$, where $N\left(\lambda x_{h}\right)$ stands for the normal form of $\lambda x_{h}$ in $\Lambda$, i.e., $N\left(\lambda x_{h}\right):=$ $x_{t_{1}} \cdots x_{t_{\mu_{\lambda}(h)}} x_{h} x_{t_{\mu_{\lambda}(h)+1}} \cdots x_{t_{j}}$.

Clearly, $N_{m}$ has a basis $\mathcal{N}_{m}:=\left\{\lambda \otimes x_{1}^{i_{1}} \cdots x_{n}^{i_{n}} \mid \lambda \in \mathcal{B}, i_{1}+i_{2}+\cdots+i_{n}=\right.$ $m\}$. If $\lambda \in \mathcal{B}_{j}$ then $j$ is called the degree of $\lambda \otimes x_{1}^{i_{1}} \cdots x_{n}^{i_{n}}$, and $j+\sum_{h=1}^{n} i_{h}$ is called the total degree of $\lambda \otimes x_{1}^{i_{1}} \cdots x_{n}^{i_{n}}$. If, viewed as a monomial in $k\left[x_{1}, \ldots, x_{n}\right]$, $\lambda x_{1}^{i_{1}} \cdots x_{n}^{i_{n}}$ can be written as $x_{1}^{j_{1}} \cdots x_{n}^{j_{n}}$, then $\left|\left\{l \mid j_{l} \neq 0,1 \leq l \leq n\right\}\right|$ is called the grade of $\lambda \otimes x_{1}^{i_{1}} \cdots x_{n}^{i_{n}}$.

First, denote by $N_{m}(i)$ the subspace of $N_{m}$ generated by all elements in $\mathcal{N}_{m}$ of grade $i$. Since $\sigma_{m}$ keeps the grade of the elements, we have rank $\sigma_{m}=$ $\sum_{i=1}^{n} \operatorname{rank}\left(\sigma_{m} \mid N_{m}(i)\right)$. 
Second, denote by $N_{m}\left(x_{s_{1}} \cdots x_{s_{i}}\right)$ the subspace of $N_{m}(i)$ generated by all elements $\lambda \otimes x_{1}^{i_{1}} \cdots x_{n}^{i_{n}}$ in $\mathcal{N}_{m}$ satisfying, viewed as monomials in $k\left[x_{1}, \ldots, x_{n}\right]$, $\lambda x_{1}^{i_{1}} \cdots x_{n}^{i_{n}}=x_{s_{1}}^{j_{1}} \cdots x_{s_{i}}^{j_{i}}$. Thus rank $\sigma_{m}=\sum_{i=1}^{n}\left(\begin{array}{c}n \\ i\end{array}\right) \operatorname{rank}\left(\sigma_{m} \mid N_{m}\left(x_{s_{1}} \cdots x_{s_{i}}\right)\right)$.

Third, denote $N_{m}\left(x_{s_{1}} \cdots x_{s_{i}}, j\right)$ the subspace of $N_{m}\left(x_{s_{1}} \cdots x_{s_{i}}\right)$ generated by all elements $\lambda \otimes x_{1}^{i_{1}} \cdots x_{n}^{i_{n}}$ in $\mathcal{N}_{m}$ of degree $j$ (equivalently, of total degree $j+m)$ with $0 \leq j \leq i$. Moreover, denote $N_{m}\left(x_{s_{1}}^{j_{1}} \cdots x_{s_{i}}^{j_{i}}, j\right)$ the subspace of $N_{m}\left(x_{s_{1}} \cdots x_{s_{i}}, j\right)$ generated by all elements $\lambda \otimes x_{1}^{i_{1}} \cdots x_{n}^{i_{n}}$ in $\mathcal{N}_{m}$ with $\lambda x_{1}^{i_{1}} \cdots x_{n}^{i_{n}}=x_{s_{1}}^{j_{1}} \cdots x_{s_{i}}^{j_{i}}, j_{1}, \ldots, j_{i} \geq 1$ and $\sum_{l=1}^{i} j_{l}=j+m$. Consider the basis $\mathcal{N}_{m}\left(x_{s_{1}}^{j_{1}} \cdots x_{s_{i}}^{j_{i}}, j\right):=\mathcal{N}_{m} \cap N_{m}\left(x_{s_{1}}^{j_{1}} \cdots x_{s_{i}}^{j_{i}}, j\right)$ of the vector space $N_{m}\left(x_{s_{1}}^{j_{1}} \cdots x_{s_{i}}^{j_{i}}, j\right)$. Order the elements $\lambda \otimes x_{1}^{i_{1}} \cdots x_{n}^{i_{n}}$ in $\mathcal{N}_{m}\left(x_{s_{1}}^{j_{1}} \cdots x_{s_{i}}^{j_{i}}, j\right)$ by the left lexicographic order on $\lambda$. Obviously $\sigma_{m}$ maps $N_{m}\left(x_{s_{1}}^{j_{1}} \cdots x_{s_{i}}^{j_{i}}, j\right)$ into $N_{m-1}\left(x_{s_{1}}^{j_{1}} \cdots x_{s_{i}}^{j_{i}}, j+1\right)$ for $0 \leq j \leq i-1$ and into 0 for $j=i$. Let $\alpha: N_{m}\left(x_{s_{1}}^{j_{1}} \cdots x_{s_{i}}^{j_{i}}, j\right) \rightarrow N_{m-1}\left(x_{s_{1}}^{j_{1}} \cdots x_{s_{i}}^{j_{i}}, j+1\right)$ with $0 \leq j \leq i-1$ be the restriction of $\sigma_{m}$. Written as a matrix under the basis $\mathcal{N}_{m}\left(x_{s_{1}}^{j_{1}} \cdots x_{s_{i}}^{j_{i}}, j\right)$ of $N_{m}\left(x_{s_{1}}^{j_{1}} \cdots x_{s_{i}}^{j_{i}}, j\right)$ and $\mathcal{N}_{m-1}\left(x_{s_{1}}^{j_{1}} \cdots x_{s_{i}}^{j_{i}}, j+1\right)$ of $N_{m-1}\left(x_{s_{1}}^{j_{1}} \cdots x_{s_{i}}^{j_{i}}, j+1\right), \alpha$ is a $\left(\begin{array}{c}i \\ j+1\end{array}\right) \times\left(\begin{array}{c}i \\ j\end{array}\right)$ matrix. Partition the elements $\lambda \otimes x_{s_{1}}^{l_{1}} \cdots x_{s_{i}}^{l_{i}}$ in $\mathcal{N}_{m}\left(x_{s_{1}}^{j_{1}} \cdots x_{s_{i}}^{j_{i}}, j\right)$ and $\mathcal{N}_{m-1}\left(x_{s_{1}}^{j_{1}} \cdots x_{s_{i}}^{j_{i}}, j+1\right)$ according to whether $\lambda$ contains $x_{s_{1}}$ or not. In this way, neglected the sign $(-1)^{j}+(-1)^{m}$, this matrix is partitioned into a $2 \times 2$ partitioned matrix $\left[\begin{array}{cc}A & I \\ 0 & B\end{array}\right]$ where $A$ is a $\left(\begin{array}{c}i-1 \\ j\end{array}\right) \times\left(\begin{array}{c}i-1 \\ j-1\end{array}\right)$ matrix, $B$ is a $\left(\begin{array}{c}i-1 \\ j+1\end{array}\right) \times\left(\begin{array}{c}i-1 \\ j\end{array}\right)$ matrix and $I$ is a $\left(\begin{array}{c}i-1 \\ j\end{array}\right) \times\left(\begin{array}{c}i-1 \\ j\end{array}\right)$ identity matrix.

Claim. $B A=0$.

Proof of the Claim. Take any row of $B$. Assume that it corresponds to the element $x_{q_{1}} \cdots x_{q_{j+1}} \in \mathcal{N}_{m-1}\left(x_{s_{1}}^{j_{1}} \cdots x_{s_{i}}^{j_{i}}, j+1\right)$. Then, according to the definition of $\sigma_{m}$, this row is just the vector whose components corresponding to $x_{q_{1}} \cdots x_{q_{j}}, \ldots, x_{q_{2}} \cdots x_{q_{j+1}}$ are $(-1)^{j}, \ldots,(-1)^{0}$ respectively, and other components are 0 . Take any column of $A$. Assume that it corresponds to the element $x_{s_{1}} x_{q_{1}} \cdots \hat{x}_{q_{a}} \cdots \hat{x}_{q_{b}} \cdots x_{q_{j+1}} \in \mathcal{N}_{m}\left(x_{s_{1}}^{j_{1}} \cdots x_{s_{i}}^{j_{i}}, j\right)$. Here $\hat{x}$ means $x$ is deleted. This column is just the vector whose components corresponding to $x_{s_{1}} x_{q_{1}} \cdots x_{q_{a}} \cdots \hat{x}_{q_{b}} \cdots x_{q_{j+1}}$ and $x_{s_{1}} x_{q_{1}} \cdots \hat{x}_{q_{a}} \cdots x_{q_{b}} \cdots x_{q_{j+1}}$ are $(-1)^{a}$ and $(-1)^{b-1}$ respectively. Thus the inner product of this row of $B$ and this column of $A$ is just $(-1)^{a}(-1)^{b-1}+(-1)^{a-1}(-1)^{b-1}=0$. Hence the claim holds.

Note that under the chosen basis $\alpha$ is the matrix $\left((-1)^{j}+(-1)^{m}\right)\left[\begin{array}{cc}A & I \\ 0 & B\end{array}\right]$. By the Claim we have

$$
\begin{aligned}
\operatorname{rank}\left(\sigma_{m} \mid N_{m}\left(x_{s_{1}}^{j_{1}} \cdots x_{s_{i}}^{j_{i}}, j\right)\right) & =\operatorname{rank} \alpha \\
& =\left\{\begin{array}{cl}
\left(\begin{array}{c}
i-1 \\
j
\end{array}\right), & \text { if } p(j)=p(m) \text { and char } k \neq 2 ; \\
0, & \text { otherwise. }
\end{array}\right.
\end{aligned}
$$


Since

$$
\operatorname{rank}\left(\sigma_{m} \mid N_{m}\left(x_{s_{1}} \cdots x_{s_{i}}\right)\right)=\sum_{j=0}^{i-1}\left(\begin{array}{c}
j+m-1 \\
i-1
\end{array}\right) \operatorname{rank}\left(\sigma_{m} \mid N_{m}\left(x_{s_{1}}^{j_{1}} \cdots x_{s_{i}}^{j_{i}}, j\right)\right),
$$

we have

$$
\begin{aligned}
\operatorname{rank} \sigma_{m} & =\sum_{i=1}^{n}\left(\begin{array}{c}
n \\
i
\end{array}\right) \sum_{j=0}^{i-1}\left(\begin{array}{c}
j+m-1 \\
i-1
\end{array}\right) \operatorname{rank}\left(\sigma_{m} \mid N_{m}\left(x_{s_{1}}^{j_{1}} \cdots x_{s_{i}}^{j_{i}}, j\right)\right) \\
& = \begin{cases}\sum_{i=1}^{n}\left(\begin{array}{c}
n \\
i
\end{array}\right) \sum_{\substack{j=0 \\
p(j)=p(m)}}^{i-1}\left(\begin{array}{c}
j+m-1 \\
i-1
\end{array}\right)\left(\begin{array}{c}
i-1 \\
j
\end{array}\right), & \text { if char } k \neq 2 ; \\
0, & \text { otherwise. }\end{cases}
\end{aligned}
$$

The lemma follows from the fact that $\operatorname{rank} \tau_{m}=\operatorname{rank} \sigma_{m}$.

Lemma 2. $\sum_{i=j+1}^{n}\left(\begin{array}{c}n \\ i\end{array}\right)\left(\begin{array}{c}j+m-1 \\ i-1\end{array}\right)\left(\begin{array}{c}i-1 \\ j\end{array}\right)=\sum_{i=1}^{n-j}\left(\begin{array}{c}n-i \\ j\end{array}\right)\left(\begin{array}{c}m+n-i-1 \\ n-i\end{array}\right)$.

Proof. For $0 \leq j \leq n-1, m \geq 1-j$ and $0 \leq r \leq i-1$, define $S_{m, r}=\sum_{i=j+1}^{n}\left(\begin{array}{c}n \\ i\end{array}\right)\left(\begin{array}{c}j+m-1 \\ i-1-r\end{array}\right)\left(\begin{array}{c}i-1 \\ j\end{array}\right)$, and $T_{m, r}=\sum_{i=1}^{n-j}\left(\begin{array}{c}n-i \\ j\end{array}\right)\left(\begin{array}{c}m+n-i-1 \\ n-i-r\end{array}\right)$. We shall show that $S_{m, r}=T_{m, r}$ for all $0 \leq r \leq n-1$ and $m \geq 1-i$.

Firstly, we have $S_{m, n-1}=T_{m, n-1}$ for all $m \geq 1-j$ : This follows from $S_{m, n-1}=\sum_{i=j+1}^{n}\left(\begin{array}{c}n \\ i\end{array}\right)\left(\begin{array}{c}j+m-1 \\ i-n\end{array}\right)\left(\begin{array}{c}i-1 \\ j\end{array}\right)=\left(\begin{array}{l}n \\ n\end{array}\right)\left(\begin{array}{c}j+m-1 \\ 0\end{array}\right)\left(\begin{array}{c}n-1 \\ j\end{array}\right)=\left(\begin{array}{c}n-1 \\ j\end{array}\right)$ and $T_{m, n-1}=$ $\sum_{i=1}^{n-j}\left(\begin{array}{c}n-i \\ j\end{array}\right)\left(\begin{array}{c}m+n-i-1 \\ 1-i\end{array}\right)=\left(\begin{array}{c}n-1 \\ j\end{array}\right)$.

Secondly, we have $S_{1-j, r}=T_{1-j, r}$ for all $0 \leq r \leq n-1$ : Note that $S_{1-j, r}=$ $\sum_{i=j+1}^{n}\left(\begin{array}{c}n \\ i\end{array}\right)\left(\begin{array}{c}0 \\ i-1-r\end{array}\right)\left(\begin{array}{c}i-1 \\ j\end{array}\right)$ and $T_{1-j, r}=\sum_{i=1}^{n-j}\left(\begin{array}{c}n-i \\ j\end{array}\right)\left(\begin{array}{c}n-i-j \\ n-i-r\end{array}\right)$. If $r<i$ then $S_{1-j, r}=0=$ $T_{1-j, r}$. If $r \geq i$ then $S_{1-j, r}=\left(\begin{array}{c}n \\ r+1\end{array}\right)\left(\begin{array}{c}r \\ j\end{array}\right)$ and $T_{1-j, r}=\sum_{i=1}^{n-j}\left(\begin{array}{c}n-i \\ r\end{array}\right)\left(\begin{array}{c}r \\ i\end{array}\right)=\left(\begin{array}{c}n \\ r+1\end{array}\right)\left(\begin{array}{c}r \\ j\end{array}\right)$.

Thirdly, we have $S_{m, r}=T_{m, r}$ for all $0 \leq r \leq n-1$ and $m \geq 1-j$ : Since $S_{m, r}=S_{m-1, r+1}+S_{m-1, r}$ and $T_{m, r}=T_{m-1, r+1}+T_{m-1, r}$, we have

$$
S_{m, r}=S_{m-1, r+1}+S_{m-2, r+1}+\cdots+S_{1-j, r+1}+S_{1-j, r}
$$

and

$$
T_{m, r}=T_{m-1, r+1}+T_{m-2, r+1}+\cdots+T_{1-j, r+1}+T_{1-j, r} .
$$

Thus

$$
\begin{aligned}
S_{m, n-2} & =S_{m-1, n-1}+S_{m-2, n-1}+\cdots+S_{1-j, n-1}+S_{1-j, n-2} \\
& =T_{m-1, n-1}+T_{m-2, n-1}+\cdots+T_{1-j, n-1}+T_{1-j, n-2} \\
& =T_{m, n-2}
\end{aligned}
$$


for all $m \geq 1-j$. Similarly, by induction, we have $S_{m, r}=T_{m, r}$ for all $0 \leq r \leq n-1$ and $m \geq 1-j$.

Fourthly and finally, Lemma 2 follows from $S_{m, 0}=T_{m, 0}$.

Lemma 3. $\operatorname{rank} \tau_{m}=\sum_{i=1}^{n-1} 2^{i-1}\left(\begin{array}{c}m+i-1 \\ i\end{array}\right)+ \begin{cases}1, & \text { if } m \text { is even; } \\ 0, & \text { if } m \text { is odd }\end{cases}$

Proof. By Lemma 2, we have

$$
\begin{aligned}
& \operatorname{rank} \tau_{m}=\sum_{\substack{j=0 \\
p(j)=p(m)}}^{n-1} \sum_{i=j+1}^{n}\left(\begin{array}{c}
n \\
i
\end{array}\right)\left(\begin{array}{c}
j+m-1 \\
i-1
\end{array}\right)\left(\begin{array}{c}
i-1 \\
j
\end{array}\right) \\
& =\sum_{\substack{j=0 \\
p(j)=p(m)}}^{n-1} \sum_{i=1}^{n-j}\left(\begin{array}{c}
n-i \\
j
\end{array}\right)\left(\begin{array}{c}
m+n-i-1 \\
n-i
\end{array}\right) \\
& =\sum_{i=1}^{n} \sum_{\substack{j=0 \\
p(j)=p(m)}}^{n-i}\left(\begin{array}{c}
n-i \\
j
\end{array}\right)\left(\begin{array}{c}
m+n-i-1 \\
n-i
\end{array}\right) \\
& =\sum_{i=1}^{n}\left(\begin{array}{c}
m+n-i-1 \\
n-i
\end{array}\right) \sum_{\substack{j=0 \\
p(j)=p(m)}}^{n-i}\left(\begin{array}{c}
n-i \\
j
\end{array}\right) \\
& =\sum_{i=1}^{n-1} 2^{n-i-1}\left(\begin{array}{c}
m+n-i-1 \\
n-i
\end{array}\right)+ \begin{cases}1, & \text { if } m \text { is even; } \\
0, & \text { if } m \text { is odd }\end{cases} \\
& =\sum_{i=1}^{n-1} 2^{i-1}\left(\begin{array}{c}
m+i-1 \\
i
\end{array}\right)+ \begin{cases}1, & \text { if } m \text { is even } \\
0, & \text { if } m \text { is odd. }\end{cases}
\end{aligned}
$$

Lemma 4. $\operatorname{rank} \tau_{m}+\operatorname{rank} \tau_{m+1}=2^{n-1}\left(\begin{array}{c}m+n-1 \\ n-1\end{array}\right)$.

Proof. Denote $U_{n}^{m}:=\sum_{i=1}^{n-1} 2^{i-1}\left(\begin{array}{c}m+i-1 \\ i\end{array}\right)$. Then $U_{n}^{m+1}=\sum_{i=1}^{n-1} 2^{i-1}\left(\begin{array}{c}m+i \\ i\end{array}\right)$ and $2 U_{n}^{m+1}=\sum_{i=1}^{n-1} 2^{i}\left(\begin{array}{c}m+i \\ i\end{array}\right)$. So $-U_{n}^{m+1}=\left(\begin{array}{c}m+1 \\ 1\end{array}\right)+\sum_{i=2}^{n-1} 2^{i-1}\left(\begin{array}{c}m+i-1 \\ i\end{array}\right)-2^{n-1}\left(\begin{array}{c}m+n-1 \\ n-1\end{array}\right)=$ $U_{n}^{m}-2^{n-1}\left(\begin{array}{c}m+n-1 \\ n-1\end{array}\right)+1$. Thus $U_{n}^{m}+U_{n}^{m+1}=2^{n-1}\left(\begin{array}{c}m+n-1 \\ n-1\end{array}\right)-1$. By Lemma 3 , we have $\operatorname{rank} \tau_{m}=U_{n}^{m}+\left\{\begin{array}{ll}1, & \text { if } m \text { is even; } \\ 0, & \text { if } m \text { is odd. }\end{array}\right.$ Thus $\operatorname{rank} \tau_{m}+\operatorname{rank} \tau_{m+1}=$ $U_{n}^{m}+\left\{\begin{array}{ll}1, & \text { if } m \text { is even; } \\ 0, & \text { if } m \text { is odd. }\end{array}+U_{n}^{m+1}+\left\{\begin{array}{ll}1, & \text { if } m+1 \text { is even; } \\ 0, & \text { if } m+1 \text { is odd. }\end{array}=2^{n-1}\left(\begin{array}{c}m+n-1 \\ n-1\end{array}\right)\right.\right.$.

Now we can calculate the Hochschild homology of the exterior algebra: 
Theorem 2. Let $\Lambda=k Q / I$ be the exterior algebra. Then

$$
\operatorname{dim}_{k} H H_{m}(\Lambda)= \begin{cases}2^{n}\left(\begin{array}{c}
n+m-1 \\
n-1
\end{array}\right), & \text { if char } k=2 \\
2^{n-1}+1, & \text { if } m=0 \text { and char } k \neq 2 \\
2^{n-1}\left(\begin{array}{c}
n+m-1 \\
n-1
\end{array}\right), & \text { if } m \geq 1 \text { and char } k \neq 2\end{cases}
$$

Proof. Case char $k \neq 2$ : If $m \geq 1$ then, by Lemma 4 ,

$$
\begin{aligned}
\operatorname{dim}_{k} H H_{m}(\Lambda) & =\operatorname{dim}_{k} \operatorname{Ker} \tau_{m}-\operatorname{dim}_{k} \operatorname{Im} \tau_{m+1} \\
& =\operatorname{dim}_{k} P_{m}-\operatorname{dim}_{k} \operatorname{Im} \tau_{m}-\operatorname{dim}_{k} \operatorname{Im} \tau_{m+1} \\
& =2^{n}\left(\begin{array}{c}
n+m-1 \\
n-1
\end{array}\right)-\operatorname{rank} \tau_{m}-\operatorname{rank} \tau_{m+1} \\
& =2^{n-1}\left(\begin{array}{c}
n+m-1 \\
n-1
\end{array}\right)
\end{aligned}
$$

If $m=0$ then $H H_{0}(\Lambda)=\Lambda /[\Lambda, \Lambda]$, where $[\Lambda, \Lambda]=\left\{\lambda_{1} \lambda_{2}-\lambda_{2} \lambda_{1} \mid \lambda_{1}, \lambda_{2} \in\right.$ $\Lambda\}$. Note that as a vector space $[\Lambda, \Lambda]$ is generated by all commutators $x_{i_{1}} \cdots x_{i_{s}} x_{j_{1}} \cdots x_{j_{t}}-x_{j_{1}} \cdots x_{j_{t}} x_{i_{1}} \cdots x_{i_{s}}=\left(1+(-1)^{s t+1}\right) x_{i_{1}} \cdots x_{i_{s}} x_{j_{1}} \cdots x_{j_{t}}$ for all $x_{i_{1}} \cdots x_{i_{s}}, x_{j_{1}} \cdots x_{j_{t}} \in \mathcal{B}$. If both $s$ and $t$ are odd then $x_{i_{1}} \cdots x_{i_{s}} x_{j_{1}} \cdots x_{j_{t}} \in$ $[\Lambda, \Lambda]$. If $r \geq 2$ is even then $r=(r-1)+1$. Thus $x_{1} \cdots x_{r} \in[\Lambda, \Lambda]$. This implies that $[\Lambda, \Lambda]$ has a basis consisting of the images of all paths of even length $(\geq 2)$. Therefore $\Lambda /[\Lambda, \Lambda]$ has a basis consisting of the images of identity and all paths of odd length. Hence $\operatorname{dim}_{k} H H_{0}(\Lambda)=1+\sum_{\substack{i=1 \\ i \text { odd }}}^{n}\left(\begin{array}{c}n \\ i\end{array}\right)=$ $2^{n-1}+1$.

Case char $k=2$ : In this case all maps in the complex $\left(M_{\bullet}, \tau_{\bullet}\right)$ are zero. Thus $H H_{m}(\Lambda) \cong \Lambda^{\left(\begin{array}{c}n+m-1 \\ n-1\end{array}\right)}$ and $\operatorname{dim}_{k} H H_{m}(\Lambda)=2^{n}\left(\begin{array}{c}n+m-1 \\ n-1\end{array}\right)$ for $m \geq 1$. Clearly $\operatorname{dim}_{k} H H_{0}(\Lambda)=\operatorname{dim}_{k} \Lambda=2^{n}$.

Denote by $H C_{m}(\Lambda)$ the $m$-th cyclic homology group of $A$ (cf. [21]). Let $h c_{m}(\Lambda):=\operatorname{dim}_{k} H C_{m}(\Lambda)$ and $h h_{m}(\Lambda):=\operatorname{dim}_{k} H H_{m}(\Lambda)$

Corollary 1. Let $\Lambda=k Q / I$ be the exterior algebra and char $k=0$. Then $h c_{m}(\Lambda)=\sum_{i=0}^{m}(-1)^{m-i} 2^{n-1}\left(\begin{array}{c}n+i-1 \\ n-1\end{array}\right)+ \begin{cases}0, & \text { if } m \text { is even; } \\ 1, & \text { if } m \text { is odd }\end{cases}$

Proof. By [21, Theorem 4.1.13], we have

$$
\left(h c_{m}(\Lambda)-h c_{m}(k)\right)=-\left(h c_{m-1}(\Lambda)-h c_{m-1}(k)\right)+\left(h h_{m}(\Lambda)-h h_{m}(k)\right) .
$$

Thus $\left(h c_{m}(\Lambda)-h c_{m}(k)\right)=\sum_{i=0}^{m}(-1)^{m-i}\left(h h_{i}(\Lambda)-h h_{i}(k)\right)$. It is well-known that $h h_{i}(k)=\left\{\begin{array}{ll}1, & \text { if } i=0 ; \\ 0, & \text { if } i \geq 1 .\end{array}\right.$ and $h c_{m}(k)=\left\{\begin{array}{ll}1, & \text { if } m \text { is even; } \\ 0, & \text { if } m \text { is odd. }\end{array}\right.$ By Theorem 2, we have $h c_{m}(\Lambda)=\sum_{i=0}^{m}(-1)^{m-i} 2^{n-1}\left(\begin{array}{c}n+i-1 \\ n-1\end{array}\right)+ \begin{cases}0, & \text { if } m \text { is even; } \\ 1, & \text { if } m \text { is odd }\end{cases}$ 


\section{Hochschild cohomology}

In this section we calculate the $k$-dimension of the Hochschild cohomological groups of the exterior algebras.

Applying the functor $\operatorname{Hom}_{\Lambda^{e}}(-, \Lambda)$ to the minimal projective bimodule resolution $\left(P_{\bullet}, \delta_{\bullet}\right)$, we have $\operatorname{Hom}_{\Lambda^{e}}\left(\left(P_{\bullet}, \delta_{\bullet}\right), \Lambda\right)=\left(P_{\bullet}^{*}, \delta_{\bullet}^{*}\right)$ where $P_{m}^{*}=$ $\operatorname{Hom}_{\Lambda^{e}}\left(P_{m}, \Lambda\right)$ and $\delta_{m}^{*}(f)=f \delta_{m}$ for any $f \in P_{m}^{*}$. It is clear that the complex $\left(P_{\bullet}^{*}, \delta_{\bullet}^{*}\right)$ is isomorphic to the complex $\left(M^{\bullet}, \tau^{\bullet}\right)$ where $M^{m}:=\Lambda^{\left(\begin{array}{c}n+m-1 \\ n-1\end{array}\right)}$ and $\tau^{m+1}: M^{m} \rightarrow M^{m+1}, \lambda e_{1^{i_{1} \cdots n^{i n}}}^{m} \mapsto \sum_{h=1}^{n}\left(x_{h} \lambda+(-1)^{m+1} \lambda x_{h}\right) e_{1^{i_{1} \cdots h^{i} h+1} \cdots n^{i_{n}}}^{m}=$ $\left(1+(-1)^{m+j+1}\right) \sum_{h=1}^{n} \mu_{\lambda}(h) N\left(x_{h} \lambda\right) e_{1^{i_{1} \ldots h^{i} h+1} \ldots n^{i_{n}}}^{m+1}$ for $\lambda \in \mathcal{B}_{j}$. Here $\left\{e_{1^{i_{1} \ldots n^{i n}}}^{m} \mid i_{1}+\right.$ $\left.\cdots+i_{n}=m\right\}$ is the standard basis of $M^{m}$.

Lemma 5. For $m \geq 0$ we have

$$
\operatorname{rank} \tau^{m+1}= \begin{cases}\sum_{i=1}^{n}\left(\begin{array}{c}
n \\
i
\end{array}\right) \sum_{\substack{j=0 \\
p(j)=p(n+m)}}^{i-1}\left(\begin{array}{c}
j+m \\
i-1
\end{array}\right)\left(\begin{array}{c}
i-1 \\
j
\end{array}\right), & \text { if char } k \neq 2 \\
0, & \text { otherwise }\end{cases}
$$

Proof. Obviously the complex $\left(M^{\bullet}, \tau^{\bullet}\right)$ is isomorphic to the complex $\left(N^{\bullet}, \sigma^{\bullet}\right)$ which is defined by $N^{m}:=\Lambda \otimes k\left[x_{1}, \ldots, x_{n}\right]_{m}$ and $\sigma^{m+1}: N^{m} \rightarrow$ $N^{m+1}, \lambda \otimes x_{1}^{i_{1}} \cdots x_{n}^{i_{n}} \mapsto \sum_{h=1}^{n}\left(x_{h} \lambda \otimes x_{1}^{i_{1}} \cdots x_{h}^{i_{h}+1} \cdots x_{n}^{i_{n}}+(-1)^{m+1} \lambda x_{h} \otimes\right.$ $\left.x_{1}^{i_{1}} \cdots x_{h}^{i_{h}+1} \cdots x_{n}^{i_{n}}\right)$. For any $\lambda=x_{t_{1}} \cdots x_{t_{j}} \in \mathcal{B}$, we have $\sigma^{m+1}\left(\lambda \otimes x_{1}^{i_{1}} \cdots x_{n}^{i_{n}}\right)$ $=\left(1+(-1)^{m+j+1}\right) \sum_{h=1}^{n}\left(\mu_{\lambda}(h) N\left(\lambda x_{h}\right) \otimes x_{1}^{i_{1}} \cdots x_{h}^{i_{h}+1} \cdots x_{n}^{i_{n}}\right)$.

Clearly, $N^{m}$ has a basis $\mathcal{N}^{m}:=\left\{\lambda \otimes x_{1}^{i_{1}} \cdots x_{n}^{i_{n}} \mid \lambda \in \mathcal{B}, i_{1}+i_{2}+\cdots+i_{n}=\right.$ $m\}$. If, viewed as a monomial in $k\left[x_{1}, \ldots, x_{n}, x_{1}^{-1}, \ldots, x_{n}^{-1}\right], \lambda x_{1}^{-i_{1}} \cdots x_{n}^{-i_{n}}$ can be written as $x_{1}^{j_{1}} \cdots x_{n}^{j_{n}}$, then the sum $i$ (resp. $-j$ ) of all the positive (resp. negative) $j_{l}$ is called the positive (resp. negative) degree of $\lambda \otimes x_{1}^{i_{1}} \cdots x_{n}^{i_{n}}$. Note that the positive $j_{l}$ must be 1 .

Denote by $N^{m}\left(x_{s_{1}} \cdots x_{s_{i}}, x_{1}^{i_{1}} \cdots x_{n}^{i_{n}}\right)$ with $0 \leq i \leq n, 0 \leq j \leq m$ and $i_{1}+$ $\cdots+i_{n}=j$, the subspace of $N^{m}$ generated by all elements $x_{s_{1}} \cdots x_{s_{i}} x_{t_{1}} \cdots x_{t_{m-j}}$ $\otimes x_{t_{1}} \cdots x_{t_{m-j}} x_{1}^{i_{1}} \cdots x_{n}^{i_{n}}$ of positive degree $i$ and negative degree $j$ in $\mathcal{N}^{m}$ with $\left\{x_{t_{1}}, \ldots, x_{t_{m-j}}\right\} \subseteq\left\{x_{1}, \ldots, x_{n}\right\} \backslash\left\{x_{s_{1}}, \ldots, x_{s_{i}}\right\}$. Clearly this subspace is 0 unless $0 \leq m-j \leq n-i$. Consider the basis $\mathcal{N}^{m}\left(x_{s_{1}} \cdots x_{s_{i}}, x_{1}^{i_{1}} \cdots x_{n}^{i_{n}}\right):=$ $\mathcal{N}^{m} \cap N^{m}\left(x_{s_{1}} \cdots x_{s_{i}}, x_{1}^{i_{1}} \cdots x_{n}^{i_{n}}\right)$ of the vector space $N^{m}\left(x_{s_{1}} \cdots x_{s_{i}}, x_{1}^{i_{1}} \cdots x_{n}^{i_{n}}\right)$. Order the elements $\lambda \otimes x_{1}^{j_{1}} \cdots x_{n}^{j_{n}}$ in $\mathcal{N}^{m}\left(x_{s_{1}} \cdots x_{s_{i}}, x_{1}^{i_{1}} \cdots x_{n}^{i_{n}}\right)$ by the left lexicographic order on $\lambda$. Obviously $\sigma^{m+1}$ maps $N^{m}\left(x_{s_{1}} \cdots x_{s_{i}}, x_{1}^{i_{1}} \cdots x_{n}^{i_{n}}\right)$ into $N^{m+1}\left(x_{s_{1}} \cdots x_{s_{i}}, x_{1}^{i_{1}} \cdots x_{n}^{i_{n}}\right)$ if $0 \leq i \leq n-1$, and into 0 if $i=n$. Let the map 
$\alpha: N^{m}\left(x_{s_{1}} \cdots x_{s_{i}}, x_{1}^{i_{1}} \cdots x_{n}^{i_{n}}\right) \rightarrow N^{m+1}\left(x_{s_{1}} \cdots x_{s_{i}}, x_{1}^{i_{1}} \cdots x_{n}^{i_{n}}\right)$ be the restriction of $\sigma^{m}$. Written as a matrix under the basis $\mathcal{N}^{m}\left(x_{s_{1}} \cdots x_{s_{i}}, x_{1}^{i_{1}} \cdots x_{n}^{i_{n}}\right)$ of $N^{m}\left(x_{s_{1}} \cdots x_{s_{i}}, x_{1}^{i_{1}} \cdots x_{n}^{i_{n}}\right)$ and $\mathcal{N}^{m+1}\left(x_{s_{1}} \cdots x_{s_{i}}, x_{1}^{i_{1}} \cdots x_{n}^{i_{n}}\right)$ of $N^{m+1}\left(x_{s_{1}} \cdots x_{s_{i}}\right.$, $\left.x_{1}^{i_{1}} \cdots x_{n}^{i_{n}}\right), \alpha$ is an $\left(\begin{array}{c}n-i \\ m-j+1\end{array}\right) \times\left(\begin{array}{c}n-i \\ m-j\end{array}\right)$ matrix. Assume that $\left\{x_{p_{1}}, \ldots, x_{p_{n-i}}\right\}=$ $\left\{x_{1}, \ldots, x_{n}\right\} \backslash\left\{x_{s_{1}}, \ldots, x_{s_{i}}\right\}$ and $x_{p_{1}}<\cdots<x_{p_{n-i}}$. Partition the elements $\lambda \otimes x_{s_{1}}^{j_{1}} \cdots x_{s_{i}}^{j_{i}}$ in $\mathcal{N}^{m}\left(x_{s_{1}} \cdots x_{s_{i}}, x_{1}^{i_{1}} \cdots x_{n}^{i_{n}}\right)$ and $\mathcal{N}^{m+1}\left(x_{s_{1}} \cdots x_{s_{i}}, x_{1}^{i_{1}} \cdots x_{n}^{i_{n}}\right)$ according to whether $\lambda$ contains $x_{p_{1}}$ or not. In this way, neglected the sign $1+(-1)^{m+(i+m-j)+1}=1+(-1)^{i-j+1}$, this matrix is partitioned into a $2 \times 2$ partitioned matrix $\left[\begin{array}{ll}A & I \\ 0 & B\end{array}\right]$ where $A$ is an $\left(\begin{array}{c}n-i-1 \\ m-j\end{array}\right) \times\left(\begin{array}{c}n-i-1 \\ m-j-1\end{array}\right)$ matrix, $B$ is an $\left(\begin{array}{c}n-i-1 \\ m-j+1\end{array}\right) \times\left(\begin{array}{c}n-i-1 \\ m-j\end{array}\right)$ matrix and $I$ is an $\left(\begin{array}{c}n-i-1 \\ m-j\end{array}\right) \times\left(\begin{array}{c}n-i-1 \\ m-j\end{array}\right)$ identity matrix. Similar to the proof of the Lemma 1, it is not difficult to show that $B A=0$. Note that, under the chosen basis, $\alpha$ is the matrix $\left(1+(-1)^{i-j+1}\right)\left[\begin{array}{cc}A & I \\ 0 & B\end{array}\right]$. Thus we have rank $\alpha= \begin{cases}\left(\begin{array}{c}n-i-1 \\ m-j\end{array}\right), & \text { if } p(i) \neq p(j) \text { and char } k \neq 2 ; \\ 0, & \text { otherwise. }\end{cases}$

Since $\sigma^{m+1}$ preserves both positive degree and negative degree, we have

$$
\operatorname{rank} \sigma^{m+1}= \begin{cases}\sum_{i=0}^{n-1}\left(\begin{array}{c}
n \\
i
\end{array}\right) \sum_{\substack{j=m-n+i \\
p(j) \neq p(i)}}^{m}\left(\begin{array}{c}
n-i+j-1 \\
n-i-1
\end{array}\right)\left(\begin{array}{c}
n-i-1 \\
m-j
\end{array}\right), & \text { if char } k \neq 2 ; \\
0, & \text { otherwise. }\end{cases}
$$

Moreover, if char $k \neq 2$ then we have

$$
\begin{aligned}
& \operatorname{rank} \sigma^{m+1}=\sum_{i=0}^{n-1}\left(\begin{array}{c}
n \\
i
\end{array}\right) \sum_{\substack{j=m-n+i \\
p(j) \neq p(i)}}^{m}\left(\begin{array}{c}
n-i+j-1 \\
n-i-1
\end{array}\right)\left(\begin{array}{c}
n-i-1 \\
m-j
\end{array}\right) \\
& =\sum_{i=1}^{n}\left(\begin{array}{c}
n \\
i
\end{array}\right) \sum_{\substack{j=0 \\
p(m-j) \neq p(n-i)}}^{i}\left(\begin{array}{c}
m-j+i-1 \\
i-1
\end{array}\right)\left(\begin{array}{c}
i-1 \\
j
\end{array}\right) \\
& =\sum_{i=1}^{n}\left(\begin{array}{c}
n \\
i
\end{array}\right) \sum_{\substack{j=0 \\
p(j) \neq p(n+m)}}^{i}\left(\begin{array}{c}
m+j-1 \\
i-1
\end{array}\right)\left(\begin{array}{c}
i-1 \\
j-1
\end{array}\right) \\
& =\sum_{i=1}^{n}\left(\begin{array}{c}
n \\
i
\end{array}\right) \sum_{\substack{j=0 \\
p(j)=p(n+m)}}^{i-1}\left(\begin{array}{c}
m+j \\
i-1
\end{array}\right)\left(\begin{array}{c}
i-1 \\
j
\end{array}\right) .
\end{aligned}
$$

The lemma follows from the fact that $\operatorname{rank} \tau^{m+1}=\operatorname{rank} \sigma^{m+1}$.

Lemma 6. $\operatorname{rank} \tau^{m}+\operatorname{rank} \tau^{m+1}=2^{n-1}\left(\begin{array}{c}n+m-1 \\ n-1\end{array}\right)$. 
Proof. Note that

$$
\begin{aligned}
& \operatorname{rank} \tau^{m+1}=\sum_{i=1}^{n}\left(\begin{array}{c}
n \\
i
\end{array}\right) \sum_{\substack{j=0 \\
p(j)=p(n+m)}}^{i-1}\left(\begin{array}{c}
j+m \\
i-1
\end{array}\right)\left(\begin{array}{c}
i-1 \\
j
\end{array}\right) \\
& =\sum_{\substack{j=0 \\
p(j)=p(n+m)}}^{n-1} \sum_{i=j+1}^{n}\left(\begin{array}{c}
n \\
i
\end{array}\right)\left(\begin{array}{c}
j+m \\
i-1
\end{array}\right)\left(\begin{array}{c}
i-1 \\
j
\end{array}\right) \\
& =\sum_{\substack{j=0 \\
p(j)=p(n+m)}}^{n-1} \sum_{i=1}^{n-j}\left(\begin{array}{c}
n-i \\
j
\end{array}\right)\left(\begin{array}{c}
m+n-i \\
n-i
\end{array}\right) \\
& =\sum_{i=1}^{n} \sum_{\substack{j=0 \\
p(j)=p(n+m)}}^{n-i}\left(\begin{array}{c}
n-i \\
j
\end{array}\right)\left(\begin{array}{c}
m+n-i \\
n-i
\end{array}\right) \\
& =\sum_{i=1}^{n}\left(\begin{array}{c}
m+n-i \\
n-i
\end{array}\right) \sum_{\substack{j=0 \\
p(j)=p(n+m)}}^{n-i}\left(\begin{array}{c}
n-i \\
j
\end{array}\right) \\
& =\sum_{i=1}^{n-1} 2^{n-i-1}\left(\begin{array}{c}
m+n-i \\
n-i
\end{array}\right)+ \begin{cases}1, & \text { if } n+m \text { is even; } \\
0, & \text { if } n+m \text { is odd. }\end{cases} \\
& =\sum_{i=1}^{n-1} 2^{i-1}\left(\begin{array}{c}
m+i \\
i
\end{array}\right)+ \begin{cases}1, & \text { if } n+m \text { is even; } \\
0, & \text { if } n+m \text { is odd. }\end{cases}
\end{aligned}
$$

where we apply Lemma 5 and Lemma 2 in the first and the third steps respectively. By the proof of Lemma 4, we have $\operatorname{rank} \tau^{m}+\operatorname{rank} \tau^{m+1}=$ $U_{n}^{m}+U_{n}^{m+1}+1=2^{n-1}\left(\begin{array}{c}n+m-1 \\ n-1\end{array}\right)$.

Theorem 3. Let $\Lambda=k Q / I$ be the exterior algebra. Then

$$
\operatorname{dim}_{k} H H^{m}(\Lambda)= \begin{cases}2^{n}\left(\begin{array}{c}
n+m-1 \\
n-1
\end{array}\right), & \text { if char } k=2 \\
2^{n-1}+1, & \text { if } m=0, n \text { is odd and char } k \neq 2 \\
2^{n-1}\left(\begin{array}{c}
n+m-1 \\
n-1
\end{array}\right), & \text { otherwise }\end{cases}
$$

Proof. Case char $k \neq 2$ : If $m \geq 1$ then, by Lemma 6 ,

$$
\begin{aligned}
\operatorname{dim}_{k} H H^{m}(\Lambda) & =\operatorname{dim}_{k} \operatorname{Ker} \tau^{m}-\operatorname{dim}_{k} \operatorname{Im} \tau^{m+1} \\
& =\operatorname{dim}_{k} P_{m}^{*}-\operatorname{dim}_{k} \operatorname{Im} \tau^{m}-\operatorname{dim}_{k} \operatorname{Im} \tau^{m+1} \\
& =2^{n}\left(\begin{array}{c}
n+m-1 \\
n-1
\end{array}\right)-\operatorname{rank} \tau^{m}-\operatorname{rank} \tau^{m+1} \\
& =2^{n-1}\left(\begin{array}{c}
n+m-1 \\
n-1
\end{array}\right) .
\end{aligned}
$$

If $m=0$ then $H H^{0}(\Lambda)=Z(\Lambda)$ which is the center of $\Lambda$. Since $\Lambda$ is gradable, $\lambda \in Z(\Lambda)$ if and only if all its components belong to $Z(\Lambda)$. Thus it is enough to consider $\mathcal{B} \cap Z(\Lambda)$. Note that $x_{t_{1}} \cdots x_{t_{i}} \in Z(\Lambda)$ if and only if $x_{t_{1}} \cdots x_{t_{i}} x_{j}=x_{j} x_{t_{1}} \cdots x_{t_{i}}$ for all $1 \leq j \leq n$, if and only if $i=n$ or $i$ is 
even. Therefore $\operatorname{dim}_{k} H H^{0}(\Lambda)=\operatorname{dim}_{k} Z(\Lambda)=\sum_{\substack{i=0 \\ i \text { even }}}^{n}\left(\begin{array}{c}n \\ i\end{array}\right)=2^{n-1}$ if $n$ is even, and $\operatorname{dim}_{k} H H^{0}(\Lambda)=2^{n-1}+1$ if $n$ is odd.

Case char $k=2$ : In these case all maps in the complex $\left(M^{\bullet}, \tau^{\bullet}\right)$ are zero. If $m \geq 1$ then $\operatorname{dim}_{k} H H^{m}(\Lambda)=\operatorname{dim}_{k} \Lambda^{\left(\begin{array}{c}n+m-1 \\ n-1\end{array}\right)}=2^{n}\left(\begin{array}{c}n+m-1 \\ n-1\end{array}\right)$. If $m=0$ then $\operatorname{dim}_{k} H H^{0}(\Lambda)=\operatorname{dim}_{k} \operatorname{Hom}_{\Lambda^{e}}\left(\Lambda^{e}, \Lambda\right)=\operatorname{dim}_{k} \Lambda=2^{n}$.

Remark 2. The case $n=2$ was obtained in [5].

Corollary 2. The Hilbert series of the exterior algebra $\Lambda$

$\sum_{m=0}^{\infty} \operatorname{dim}_{k} H H^{m}(\Lambda) t^{m}= \begin{cases}\frac{2^{n-1}}{(1-t)^{n}}, & \text { if } n \text { is even and char } k \neq 2 \\ \frac{2^{n-1}}{(1-t)^{n}}+1, & \text { if } n \text { is odd and char } k \neq 2 \\ \frac{2^{n}}{(1-t)^{n}}, & \text { if char } k=2 .\end{cases}$

Proof. Note that $\sum_{m=0}^{\infty}\left(\begin{array}{c}n+m-1 \\ n-1\end{array}\right) t^{m}=\frac{1}{(1-t)^{n}}$.

\section{Hochschild cohomology rings}

In this section we shall determine the Hochschild cohomology rings of the exterior algebras by generators and relations.

Now we construct another minimal projective bimodule resolution $\left(P_{\bullet}^{\prime}, \delta_{\bullet}^{\prime}\right)$

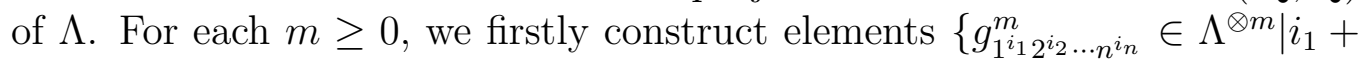
$\cdots+i_{n}=m$ with $\left.\left(i_{1}, \ldots, i_{n}\right) \in \mathbb{N}^{n}\right\}$ : Let $g_{0}^{0}=1, g_{1}^{1}=x_{1}, g_{2}^{1}=x_{2}, \ldots, g_{n}^{1}=x_{n}$. Define $g_{1^{i_{1}} 2^{i_{2} \ldots n^{i_{n}}}}^{m}$ for all $m \geq 2$ inductively by $g_{1^{i_{1}} 2^{i_{2} \ldots n^{i_{n}}}}^{m}=\sum_{h=1}^{n} g_{1^{i_{1} \cdots h^{i_{h}-1} \cdots n^{i_{n}}}}^{m-1} \otimes$ $x_{h}$, where $i_{1}+i_{2}+\cdots+i_{n}=m$ with $i_{h} \geq 0$ and $g_{1^{i_{1} \ldots h^{-1} \cdots n^{i n}}}^{m-1}=0$ for all $1 \leq h \leq n$. It is easy to see that $g_{1^{i_{1} 2_{2} \ldots n_{n}}}^{m}=\sum_{h=1}^{n} x_{h} \otimes g_{1^{i_{1} \ldots h^{i_{h}-1} \cdots n^{i_{n}}}}^{m-1}$.

Let $P_{m}^{\prime}:=\coprod_{i_{1}+i_{2}+\cdots+i_{n}=m} \Lambda \otimes g_{1^{i_{1} 2^{2} i_{2} \cdots n^{i_{n}}}} \otimes \Lambda \subseteq \Lambda^{\otimes m+2}$ for $m \geq 0$, and let

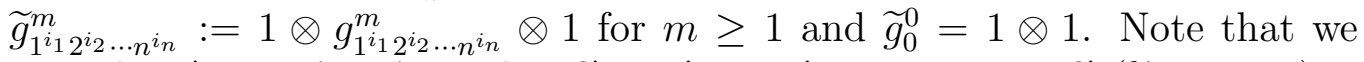
identify $P_{0}^{\prime}$ with $\Lambda \otimes \Lambda$. Define $\delta_{m}^{\prime}: P_{m}^{\prime} \rightarrow P_{m-1}^{\prime}$ by setting $\delta_{m}^{\prime}\left(\widetilde{g}_{1^{i_{1}} 2^{i_{2} \ldots n^{i n}}}^{m}\right)=$ $\sum_{h=1}^{n}\left(x_{h} \widetilde{g}_{1^{i_{1} \ldots h^{i} h^{-1} \cdots n^{i n}}}^{m-1}+(-1)^{m} \widetilde{g}_{1^{i_{1} \ldots h^{i} h^{-1} \cdots n^{i n}}}^{m-1} x_{h}\right)$.

Lemma 7. The complex $\mathbb{P}:=\left(P_{\bullet}^{\prime}, \delta_{\bullet}^{\prime}\right)$ :

$$
\cdots \rightarrow P_{m+1}^{\prime} \stackrel{\delta_{m+1}^{\prime}}{\longrightarrow} P_{m}^{\prime} \stackrel{\delta_{m}^{\prime}}{\longrightarrow} \cdots \stackrel{\delta_{3}^{\prime}}{\longrightarrow} P_{2}^{\prime} \stackrel{\delta_{2}^{\prime}}{\longrightarrow} P_{1}^{\prime} \stackrel{\delta_{1}^{\prime}}{\longrightarrow} P_{0}^{\prime} \longrightarrow 0
$$

is a minimal projective bimodule resolution of the exterior algebra $\Lambda=k Q / I$. 
Proof. Clearly the complex $\mathbb{P}=\left(P_{\bullet}^{\prime}, \delta_{\bullet}^{\prime}\right)$ is isomorphic to the complex $\left(P_{\bullet}, \delta_{\bullet}\right)$.

Applying the functor $\operatorname{Hom}_{\Lambda^{e}}(-, \Lambda)$, we have $\mathbb{P}^{*}=\left(P_{\bullet}^{\prime}, \delta_{\bullet}^{\prime}\right)^{*} \cong\left(P_{\bullet}, \delta_{\bullet}\right)^{*}=$ $\left(P_{\bullet}^{*}, \delta_{\bullet}^{*}\right) \cong\left(M^{\bullet}, \tau^{\bullet}\right)$. Thus every element in $P_{m}^{\prime *}$ can be represented as a linear

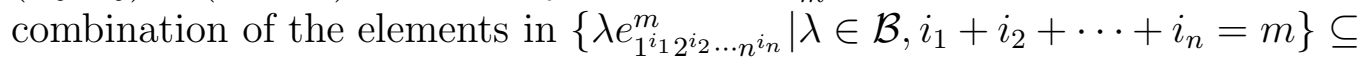
$M^{m}$. Throughout we do not distinguish an element in $\operatorname{Ker} \delta_{m+1}^{\prime *} \subseteq P_{m}^{\prime *}$ with its equivalent class in $H H^{m}(\Lambda)$. For convenience we identify $e_{i^{1} i^{1}}^{2}$ with $e_{i^{2}}^{2}$.

Lemma 8. Let $\eta=\sum_{i_{1}+i_{2}+\cdots+i_{n}=s} \lambda_{i_{1} i_{2} \cdots i_{n}} e_{1^{i_{1}} 2^{i_{2} \cdots n^{i_{n}}}}^{s} \in H H^{s}(\Lambda)$ and $\theta=$ $\sum_{j_{1}+j_{2}+\cdots+j_{n}=t} \lambda_{j_{1} j_{2} \cdots j_{n}}^{\prime} e_{1^{j_{1} 2^{j_{2} \cdots n^{j}}}}^{t} \in H H^{t}(\Lambda)$. Then the cup product of $\eta$ and $\theta$ in $H H^{s+t}(\Lambda)$ :

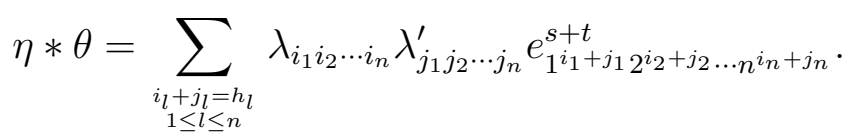

Proof. Recall that the bar resolution $\mathbb{B}=\left(B_{\bullet}, b_{\bullet}\right)$ of $\Lambda$ is given by $B_{m}:=\Lambda^{\otimes m+2}$ and $b_{m}: B_{m} \rightarrow B_{m-1}, \lambda_{0} \otimes \lambda_{1} \otimes \cdots \otimes \lambda_{m+1} \mapsto \sum_{i=0}^{m}(-1)^{i} \lambda_{0} \otimes$ $\cdots \otimes \lambda_{i-1} \otimes \lambda_{i} \lambda_{i+1} \otimes \lambda_{i+2} \otimes \cdots \otimes \lambda_{m+1}$ (cf. [21]). Define $d_{i}: B_{m} \rightarrow$ $B_{m-1}, \lambda_{0} \otimes \lambda_{1} \otimes \cdots \otimes \lambda_{m+1} \mapsto \lambda_{0} \otimes \cdots \otimes \lambda_{i-1} \otimes \lambda_{i} \lambda_{i+1} \otimes \lambda_{i+2} \otimes \cdots \otimes \lambda_{m+1}$ with $i=0,1, \ldots, m$. Then $b_{m}=\sum_{i=0}^{m}(-1)^{i} d_{i}$. View $P_{m}^{\prime}$ as a submodule of $B_{m}$ in the natural way. The complex $\mathbb{P}$ is a subcomplex of $\mathbb{B}$ : Indeed, for any given

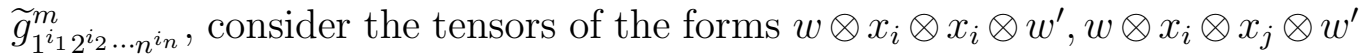
or $w \otimes x_{j} \otimes x_{i} \otimes w^{\prime}$ occurring in $\widetilde{g}_{1^{i_{1}} 2^{i_{2} \ldots n^{i n}}}^{m}$, where $w$ is a $t$-fold tensor and $w^{\prime}$ is an $(m-t)$-fold tensor. Clearly, $d_{t}$ maps $w \otimes x_{i} \otimes x_{i} \otimes w^{\prime}$ to 0 for all $1 \leq t \leq m-1$. If a tensor $w \otimes x_{i} \otimes x_{j} \otimes w^{\prime}$ occurs in $\widetilde{g}_{1^{i_{1} 2_{2} \ldots \ldots n_{n}}}^{m}$ then by defini-

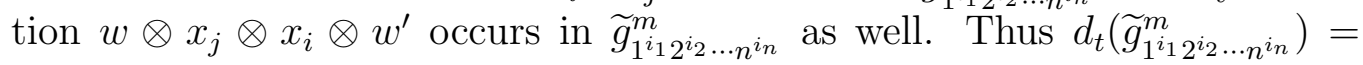
0 for all $1 \leq t \leq m-1$. Since $g_{1^{i_{1}} 2^{i_{2} \ldots n^{i_{n}}}}^{m}=\sum_{h=1}^{n} g_{1^{i_{1} \ldots h^{i_{h}}-1} \cdots n^{i_{n}}}^{m-1} \otimes x_{h}=$ $\sum_{h=1}^{n} x_{h} \otimes g_{1^{i_{1} \ldots h^{i} h^{-1} \ldots n^{i_{n}}}}^{m-1}$, we have $b_{m}\left(\widetilde{g}_{1^{i_{1}} 2^{i_{2} \ldots n^{i_{n}}}}^{m}\right)=\sum_{i=0}^{m}(-1)^{i} d_{i}\left(\widetilde{g}_{1^{i_{1}} 2^{i_{2} \cdots n^{i_{n}}}}^{m}\right)=$ $d_{0}\left(\widetilde{g}_{1^{i_{1}} 2^{i_{2} \ldots n^{i_{n}}}}^{m}\right)+(-1)^{m} d_{m}\left(\widetilde{g}_{1^{i_{1}} 2^{i_{2} \cdots n^{i_{n}}}}^{m}\right)=\delta_{m}^{\prime}\left(\widetilde{g}_{1^{i_{1}} 2^{i_{2} \cdots n^{i_{n}}}}^{m}\right)$.

Consider the diagonal map $\Delta: \mathbb{B} \rightarrow \mathbb{B} \otimes_{\Lambda} \mathbb{B}$ given by $\Delta\left(\lambda_{0} \otimes \cdots \otimes \lambda_{m+1}\right)=$ $\sum_{i=0}^{m}\left(\lambda_{0} \otimes \cdots \otimes \lambda_{i} \otimes 1\right) \otimes_{\Lambda}\left(1 \otimes \lambda_{i+1} \otimes \cdots \otimes \lambda_{m+1}\right)$. The cup product $\eta^{\prime} \cup \theta^{\prime}$ in the Hochschild cohomology ring $H H^{*}(\Lambda)$ of two cycles $\eta^{\prime}$ and $\theta^{\prime}$ from $\operatorname{Hom}_{\Lambda^{e}}(\mathbb{B}, \Lambda)$ is given by the composition $\mathbb{B} \stackrel{\Delta}{\longrightarrow} \mathbb{B} \otimes_{\Lambda} \mathbb{B} \stackrel{\eta^{\prime} \otimes \theta^{\prime}}{\longrightarrow} \Lambda \otimes_{\Lambda} \Lambda \stackrel{\nu}{\longrightarrow} \Lambda$ where $\nu: \Lambda \otimes_{\Lambda} \Lambda \rightarrow \Lambda$ is the multiplication in $\Lambda$ (cf. [24]). 
Let $\mu: \mathbb{P} \rightarrow \mathbb{B}$ be the natural inclusion and let $\pi: \mathbb{B} \rightarrow \mathbb{P}$ be a chain map such that $\pi \mu=1_{\mathbb{P}}$. We have $\Delta(\mu \mathbb{P}) \subseteq \mu \mathbb{P} \otimes_{\Lambda} \mu \mathbb{P} \subseteq \mathbb{B} \otimes_{\Lambda} \mathbb{B}$ : Indeed, fix an $s$ with $0 \leq s \leq m$, it is easy to see that $g_{1^{h_{1}} 2^{h_{2} \cdots n^{h_{n}}}}^{m}=$ $\sum_{\substack{i_{l}+j_{l}=h_{l} \\ 1 \leq l \leq n}} g_{1^{i_{1} 2_{2} \ldots} n_{n_{n}}}^{s} g_{1^{j_{1} 2^{j_{2} \ldots n^{j}}}}^{m-s}$, here we neglect $g_{0}^{0}$ if $s=0$ or $m$. Thus we can infer that $\Delta\left(\widetilde{g}_{1^{h_{1}} 2^{h_{2} \ldots n^{h_{n}}}}^{m}\right)=\sum_{s=0}^{m} \sum_{\substack{i_{l}+j_{l}=h_{l} \\ 1 \leq l \leq n}} \widetilde{g}_{1^{i_{1}} 2^{i_{2} \ldots n^{i n}}}^{s} \widetilde{g}_{1^{j_{1}} 2^{j_{2} \ldots n^{j n}}}^{m-s}$ which implies that $\Delta(\mu \mathbb{P}) \subseteq \mu \mathbb{P} \otimes_{\Lambda} \mu \mathbb{P}$.

Let $\Delta^{\prime}$ be the restriction of $\Delta$ on $\mathbb{P}$. Then $\Delta \mu=(\mu \otimes \mu) \Delta^{\prime}$. Viewed as elements in $P_{s}^{\prime *}$ and $P_{t}^{\prime *}, \eta$ and $\theta$ can be represented by $\eta \pi_{s}$ and $\theta \pi_{t}$ by the bar resolution respectively. Therefore

$$
\begin{aligned}
\eta * \theta & =(\eta * \theta) \pi_{s+t} \mu_{s+t} \\
& =\left(\eta \pi_{s} \cup \theta \pi_{t}\right) \mu_{s+t} \\
& =\nu\left(\eta \pi_{s} \otimes \theta \pi_{t}\right) \Delta \mu_{s+t} \\
& =\nu\left(\eta \pi_{s} \otimes \theta \pi_{t}\right)\left(\mu_{s} \otimes \mu_{t}\right) \Delta^{\prime} \\
& =\nu(\eta \otimes \theta) \Delta^{\prime}
\end{aligned}
$$

Since $\eta\left(\widetilde{g}_{1^{i_{1}} 2^{i_{2} \cdots n^{i_{n}}}}^{s}\right)=\lambda_{i_{1} i_{2} \cdots i_{n}}$ and $\theta\left(\widetilde{g}_{1^{j_{1} 2^{j_{2} \cdots n^{j}}}}^{t}\right)=\lambda_{j_{1} j_{2} \cdots j_{n}}^{\prime}$, we have

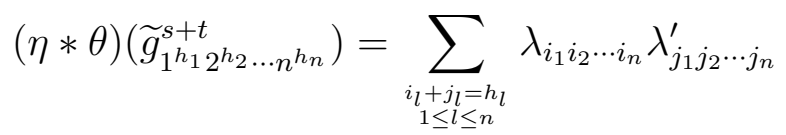

for all $\left(h_{1}, \ldots, h_{n}\right) \in \mathbb{N}^{n}$ with $h_{1}+\cdots+h_{n}=s+t$. Viewed as an element in $M^{s+t}$

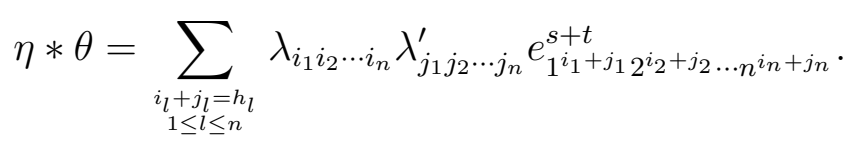

Lemma 9. Let $\Lambda=k Q / I$ be the exterior algebra and char $k \neq 2$. Then the $k$-vector space $H H^{m}(\Lambda)$ has a basis $\left\{\lambda e_{1^{i_{1}} 2^{i_{2} \ldots n^{i_{n}}}} \mid \lambda \in \mathcal{B}_{i}, p(i)=p(m), 1 \leq\right.$ $i \leq n\}$.

Proof. If $\lambda \in \mathcal{B}_{i}$ and $p(i)=p(m)$ then $\delta_{m+1}^{*}\left(\lambda e_{1^{i_{1} 2^{i_{2} \ldots n^{i n}}}}^{m}\right)=0$. Thus

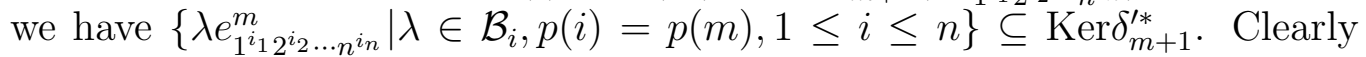

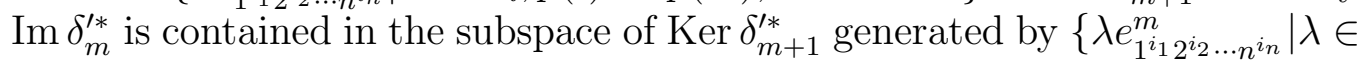
$\left.\mathcal{B}_{i}, p(i) \neq p(m), 1 \leq i \leq n\right\}$. By Theorem 3, we have $\operatorname{dim}_{k} H H^{m}(\Lambda)=$ $2^{n-1}\left(\begin{array}{c}n+m-1 \\ n-1\end{array}\right)$. Thus the Lemma holds.

As a $k$-algebra, $H H^{*}(\Lambda)$ is generated by $\left\{x_{i} x_{j} \mid 1 \leq i<j \leq n\right\} \cup\left\{x_{p} e_{q^{1}}^{1} \mid 1 \leq\right.$ $p, q \leq n\} \cup\left\{e_{s t}^{2} \mid 1 \leq s \leq t \leq n\right\}$ which satisfy all relations in the following Table H. 


\section{Table H}
(H1.1) $\quad\left(x_{i} x_{j}\right)\left(x_{s} x_{t}\right)=\left(x_{s} x_{t}\right)\left(x_{i} x_{j}\right)$
(H1.2) $\quad\left(x_{i} x_{j}\right)\left(x_{s} x_{t}\right)=0$
if $\{i, j\} \cap\{s, t\} \neq \emptyset$
(H1.3) $\quad\left(x_{i} x_{j}\right)\left(x_{s} x_{t}\right)=-\left(x_{i} x_{s}\right)\left(x_{j} x_{t}\right)$
if $i<s<j<t$
(H1.4) $\quad\left(x_{i} x_{j}\right)\left(x_{s} x_{t}\right)=\left(x_{i} x_{s}\right)\left(x_{t} x_{j}\right)$
if $i<s<t<j$
$(H 1.5) \quad\left(x_{i} x_{j}\right)\left(x_{s} x_{t}\right)=-\left(x_{s} x_{i}\right)\left(x_{t} x_{j}\right)$
if $s<i<t<j$
(H1.6) $\quad\left(x_{i} x_{j}\right)\left(x_{s} x_{t}\right)=\left(x_{s} x_{i}\right)\left(x_{j} x_{t}\right)$
if $s<i<j<t$
(H2.1) $\quad\left(x_{i} x_{j}\right)\left(x_{s} e_{t^{1}}^{1}\right)=\left(x_{s} e_{t^{1}}^{1}\right)\left(x_{i} x_{j}\right)$
$(H 2.2) \quad\left(x_{i} x_{j}\right)\left(x_{s} e_{t^{1}}^{1}\right)=0$
if $s \in\{i, j\}$
(H2.3) $\quad\left(x_{i} x_{j}\right)\left(x_{s} e_{t^{1}}^{1}\right)=\left(x_{s} x_{i}\right)\left(x_{j} e_{t^{1}}^{1}\right) \quad$ if $s<i<j$
(H2.4) $\quad\left(x_{i} x_{j}\right)\left(x_{s} e_{t^{1}}^{1}\right)=-\left(x_{i} x_{s}\right)\left(x_{j} e_{t^{1}}^{1}\right) \quad$ if $i<s<j$
$(H 3.1) \quad\left(x_{i} x_{j}\right) e_{s^{1} t^{1}}^{2}=e_{s^{1} t^{1}}^{2}\left(x_{i} x_{j}\right)$
(H4.1) $\quad\left(x_{i} e_{j^{1}}^{1}\right)\left(x_{s} e_{t^{1}}^{1}\right)=0$
if $i=s$
$(H 4.2) \quad\left(x_{i} e_{j^{1}}^{1}\right)\left(x_{s} e_{t^{1}}^{1}\right)=\left(x_{i} x_{s}\right) e_{j^{1} t^{1}}^{2}$
if $i<s$ and $j \leq t$
(H4.3) $\quad\left(x_{i} e_{j^{1}}^{1}\right)\left(x_{s} e_{t^{1}}^{1}\right)=\left(x_{i} x_{s}\right) e_{t^{1} j^{1}}^{2}$ if $i<s$ and $t \leq j$
(H4.4) $\quad\left(x_{i} e_{j^{1}}^{1}\right)\left(x_{s} e_{t^{1}}^{1}\right)=-\left(x_{s} x_{i}\right) e_{j^{1} t^{1}}^{2} \quad$ if $s<i$ and $j \leq t$
(H4.5) $\quad\left(x_{i} e_{j^{1}}^{1}\right)\left(x_{s} e_{t^{1}}^{1}\right)=-\left(x_{s} x_{i}\right) e_{t^{1} j^{1}}^{2} \quad$ if $s<i$ and $t \leq j$
(H5.1) $\quad\left(x_{i} e_{j^{1}}^{1}\right) e_{s^{1} t^{1}}^{2}=e_{s^{1} t^{1}}^{2}\left(x_{i} e_{j^{1}}^{1}\right)$
$(H 5.2) \quad\left(x_{i} e_{j^{1}}^{1}\right) e_{s^{1} t^{1}}^{2}=\left(x_{i} e_{s^{1}}^{1}\right) e_{j^{1} t^{1}}^{2}$
$(H 5.3) \quad\left(x_{i} e_{j^{1}}^{1}\right) e_{s^{1} t^{1}}^{2}=\left(x_{i} e_{s^{1}}^{1}\right) e_{t^{1} j^{1}}^{2}$
if $s<j \leq t$
if $s<t \leq j$
(H6.1) $e_{i^{1} j^{1}}^{2} e_{s^{1} t^{1}}^{2}=e_{s^{1} t^{1}}^{2} e_{i^{1} j^{1}}^{2}$
(H6.2) $e_{i^{1} j^{1}}^{2} e_{s^{1} t^{1}}^{2}=e_{i^{1} s^{1}}^{2} e_{j^{1} t^{1}}^{2}$
(H6.3) $e_{i^{1} j^{1}}^{2} e_{s^{1} t^{1}}^{2}=e_{i^{1} s^{1}}^{2} e_{t^{1} j^{1}}^{2}$
if $i \leq s \leq j \leq t$
(H6.4) $e_{i^{1} j^{1}}^{2} e_{s^{1} t^{1}}^{2}=e_{s^{1} i^{1}}^{2} e_{t^{1} j^{1}}^{2}$
if $i \leq s \leq t \leq j$
(H6.5) $e_{i^{1} j^{1}}^{2} e_{s^{1} t^{1}}^{2}=e_{s^{1} i^{1}}^{2} e_{j^{1} t^{1}}^{2}$
if $s \leq i \leq t \leq j$
if $s \leq i \leq j \leq t$

Replace $x_{i} x_{j}, x_{p} e_{q^{1}}^{1}, e_{s^{1} t^{1}}^{2}$ with $u_{i j}, v_{p q}, w_{s t}$ respectively, we have the following Table F. 


\section{Table F}
(F1.1) $\quad u_{i j} u_{s t}=u_{s t} u_{i j}$
(F1.2) $\quad u_{i j} u_{s t}=0$
if $\{i, j\} \cap\{s, t\} \neq \emptyset$
(F1.3) $u_{i j} u_{s t}=-u_{i s} u_{j t}$
if $i<s<j<t$
(F1.4) $u_{i j} u_{s t}=u_{i s} u_{t j}$
if $i<s<t<j$
$(F 1.5)$
$u_{i j} u_{s t}=-u_{s i} u_{t j}$
if $s<i<t<j$
(F1.6)
$u_{i j} u_{s t}=u_{s i} u_{j t} \quad$ if $s<i<j<t$
$(F 2.1) \quad u_{i j} v_{s t}=v_{s t} u_{i j}$
$(F 2.2) \quad u_{i j} v_{s t}=0$
if $s \in\{i, j\}$
(F2.3) $u_{i j} v_{s t}=u_{s i} v_{j t} \quad$ if $s<i<j$
(F2.4) $u_{i j} v_{s t}=-u_{i s} v_{j t} \quad$ if $i<s<j$
(F3.1) $u_{i j} w_{s t}=w_{s t} u_{i j}$
$\begin{array}{lll}(F 4.1) & v_{i j} v_{s t}=0 & \text { if } i=s \\ (F 4.2) & v_{i j} v_{s t}=u_{i s} w_{j t} & \text { if } i<s \text { and } j \leq t \\ (F 4.3) & v_{i j} v_{s t}=u_{i s} w_{t j} & \text { if } i<s \text { and } t \leq j \\ (F 4.4) & v_{i j} v_{s t}=-u_{s i} w_{j t} & \text { if } s<i \text { and } j \leq t \\ (F 4.5) & v_{i j} v_{s t}=-u_{s i} w_{t j} & \text { if } s<i \text { and } t \leq j\end{array}$
$(F 5.1) \quad v_{i j} w_{s t}=w_{s t} v_{i j}$
$(F 5.2) \quad v_{i j} w_{s t}=v_{i s} w_{j t} \quad$ if $s<j \leq t$
(F5.3) $v_{i j} w_{s t}=v_{i s} w_{t j} \quad$ if $s<t \leq j$
(F6.1) $\quad w_{i j} w_{s t}=w_{s t} w_{i j}$
(F6.2) $\quad w_{i j} w_{s t}=w_{i s} w_{j t} \quad$ if $i \leq s \leq j \leq t$
(F6.3) $\quad w_{i j} w_{s t}=w_{i s} w_{t j} \quad$ if $i \leq s \leq t \leq j$
(F6.4) $\quad w_{i j} w_{s t}=w_{s i} w_{t j} \quad$ if $s \leq i \leq t \leq j$
(F6.5) $\quad w_{i j} w_{s t}=w_{s i} w_{j t} \quad$ if $s \leq i \leq j \leq t$

Theorem 4. Let $Q^{\prime}$ be the quiver with one vertex 1 and $2 n^{2}$ loops $\left\{u_{i j} \mid 1 \leq\right.$ $i<j \leq n\} \cup\left\{v_{p q} \mid 1 \leq p, q \leq n\right\} \cup\left\{e_{s t}^{2} \mid 1 \leq s \leq t \leq n\right\}$. Let $I^{\prime}$ be the ideal of $k Q^{\prime}$ generated by all relations in Table $F$. Then the Hochschild cohomology ring of the exterior algebra $\Lambda=k Q / I$,

$$
H H^{*}(\Lambda)= \begin{cases}k Q^{\prime} / I^{\prime}, & \text { if char } k \neq 2 \\ \Lambda\left[z_{1}, \ldots, z_{n}\right], & \text { if } \operatorname{char} k=2\end{cases}
$$

Proof. Case char $k \neq 2$ : Firstly, as a $k$-algebra $H H^{*}(\Lambda)$ is generated by $\left\{x_{i} x_{j} \mid 1 \leq i<j \leq n\right\} \cup\left\{x_{p} e_{q^{1}}^{1} \mid 1 \leq p, q \leq n\right\} \cup\left\{e_{s t}^{2} \mid 1 \leq s \leq t \leq n\right\}$. 
Thus we have an epimorphism of $k$-algebras $\psi: k Q^{\prime} \rightarrow H H^{*}(\Lambda)$ which maps $u_{i j}, v_{p q}, w_{s t}$ to $x_{i} x_{j}, x_{p} e_{q^{1}}^{1}, e_{s^{1} t^{1}}^{2}$ respectively. Comparing the relations in Table $\mathrm{H}$ with those in Table $\mathrm{F}$, we have $I^{\prime} \subseteq \operatorname{Ker} \psi$. Hence $\psi$ induces an epimorphism $\varphi: k Q^{\prime} / I^{\prime} \rightarrow H H^{*}(\Lambda)$.

Apply the relations in Table $\mathrm{H}$ one by one, it is not difficult to see that the set $\left\{\left(x_{i_{1}} x_{i_{2}}\right) \cdots\left(x_{i_{2 l-1}} x_{i_{2 l}}\right) e_{j_{1}^{1} j_{2}^{1}}^{2} \cdots e_{j_{2 r-1}^{1} j_{2 r}^{1}}^{2} \mid 1<i_{1}<i_{2}<\cdots<i_{2 l-1}<\right.$ $\left.i_{2 l}, j_{1} \leq j_{2} \leq \cdots \leq j_{2 r}\right\} \cup\left\{\left(x_{i_{1}} x_{i_{2}}\right) \cdots\left(x_{i_{2 l-1}} x_{i_{2 l}}\right)\left(x_{2 l+1} e_{j_{1}^{1}}^{1}\right) e_{j_{2}^{1} j_{3}^{1}}^{2} \cdots e_{j_{2 r}^{1} j_{2 r+1}^{1}}^{2} \mid 1<\right.$ $\left.i_{1}<i_{2}<\cdots<i_{2 l-1}<i_{2 l}<i_{2 l+1}, j_{1} \leq j_{2} \leq \cdots \leq j_{2 r} \leq j_{2 r+1}\right\}$ is a $k$-basis of $H H^{*}(\Lambda)$.

Apply the relations in Table $\mathrm{F}$ one by one, it is not difficult to see that the set $\left\{u_{i_{1} i_{2}} \cdots u_{i_{2 l-1} i_{2 l}} w_{j_{1} j_{2}} \cdots w_{j_{2 r-1} j_{2 r}} \mid 1<i_{1}<i_{2}<\cdots<i_{2 l-1}<i_{2 l}, j_{1} \leq\right.$ $\left.j_{2} \leq \cdots \leq j_{2 r}\right\} \cup\left\{u_{i_{1} i_{2}} \cdots u_{i_{2 l-1} i_{2 l}} v_{(2 l+1) j_{1}} w_{j_{2} j_{3}} \cdots w_{j_{2 r} j_{2 r+1}} \mid 1<i_{1}<i_{2}<\cdots<\right.$ $\left.i_{2 l-1}<i_{2 l}<i_{2 l+1}, j_{1} \leq j_{2} \leq \cdots \leq j_{2 r} \leq j_{2 r+1}\right\}$ is a $k$-basis of $k Q^{\prime} / I^{\prime}$.

Since $\varphi$ maps a basis element to a basis element, $\varphi$ is also a monomorphism. Hence it is an isomorphism.

Case char $k=2$ : The differential in the complex $\operatorname{Hom}_{\Lambda^{e}}(\mathbb{P}, \Lambda)$ is 0 . All maps in $\operatorname{Hom}_{\Lambda^{e}}(\mathbb{P}, \Lambda)$ represent nonzero elements in $H H^{*}(\Lambda)$. Let $\phi$ : $\Lambda\left[z_{1}, \ldots, z_{n}\right] \rightarrow H H^{*}(\Lambda), z_{i} \mapsto e_{i^{1}}^{1}$. Then $\phi$ is surjective and thus it is injective restricted to each degree. Hence it is an isomorphism.

Remark 3. The case $n=2$ was obtained in [5].

\section{References}

[1] I. Assem and J.A. de la Peña, The foundamental groups of a triangular algebra, Comm. Algebra 24 (1996), 187-208.

[2] M. Auslander, I. Reiten and S.O. SmalØ, Representation theory of artin algebras, Cambridge studies in advanced mathematics 36, Cambridge university press, Cambridge, 1995.

[3] L.L. Avramov and M. Vigueé-Poirrier, Hochschild homology criteria for smoothness, Internat. Math. Research Notices 1 (1992), 17-25.

[4] A. Beilinson, V. Ginsburg and W. Soergel, Koszul duality patterns in representation theory, J. Amer. Math. Soc. 9 (1996), 473-527.

[5] R.O. Buchweitz, E.L. Green, D. Madsen and Ø. Solberg, Finite Hochschild cohomology without finite global dimension, Preprint.

[6] M.C.R. Butler and A.D. King, Minimal resolutions of algebras, J. Algebra 212 (1999), $323-362$.

[7] C. Cibils, Cohomology of incidence algebras and simplicial complexes, J. Pure Appl. Algebra, 56 (1989), 221-232. 
[8] C. Cibils, On the Hochschild cohomology of finite-dimensional algebras, Comm. Algebra 16 (1988), 645-649.

[9] C. Cibils, 2-nilpotent and rigid finite-dimensional algebras, J. London Math. Soc. 36 (1987), 211-218.

[10] C. Cibils, Rigid monomial algebras, Math. Ann. 289 (1991), 95-109.

[11] C. Cibils, Rigidity of truncated quiver algebras, Adv. Math. 79 (1990), 18-42.

[12] M. Gerstenhaber, On the deformation of rings and algebras, Ann. Math. 79 (1964), $59-103$

[13] M. Gerstenhaber and S.P. Schack, Simplicial homology is Hochschild cohomology, J. Pure Appl. Algebra 30 (1983), 143-156.

[14] Y. Han, Hochschild (co)homology dimension, arXiv: math. RA/0408402.

[15] Y. Han and Y.G. Xu, Hochschild cohomology of special biserial algebras, Preprint.

[16] D. Happel, Hochschild cohomology of finite-dimensional algebras, Lecture Notes in Mathematics, Vol. 1404, Springer, Berlin, 1989, pp. 108-126.

[17] K. Igusa, Notes on the no loop conjecture, J. Pure Appl. Algebra 69 (1990), 161-176.

[18] B. Keller, Invariance and localization for cyclic homology of DG algebras, J. Pure Appl. Algebra 123 (1998), 223-273.

[19] S.X. Liu and P. Zhang, Hochschild homology of truncated algebras, Bull. London Math. Soc. 26 (1994), 427-430.

[20] A.C. Locateli, Hochschild cohomology of truncated quiver algebras, Comm. Algebra 27 (1999), 645-664

[21] J.L. Loday, Cyclic homology, Grundlehren 301, Springer, Berlin, 1992.

[22] S. Mac Lane, Homology, Grundlehren 114, Third corrected printing, Springer-Verlag, 1975.

[23] H. Matsumura, Commutative ring theory, Translated by M. Reid, Cambridge Univ. Press, 1986.

[24] K. Sanada, On the Hochschild cohomology of cross products, Comm. Algebra 21 (1993), 2727-2748.

[25] E. Sköldberg, The Hochschild homology of truncated and quadratic monomial algebras, J. London Math. Soc. 59 (1999), 76-86.

[26] A. Skowroński, Simply connected algebras and Hochschild cohomology, Proc. ICRA IV (Ottawa, 1992), Can. Math. Soc. Proc. Vol. 14, 1993, 431-447.

[27] Y.G. Xu, On the first Hochschild cohomology of trivial extensions of special biserial algebras, Sci. China (Ser. A) 47 (2004), 578-592.

[28] D. Zacharia. Hochschild homology of quasi-hereditary algebras. Canad. Math. Soc. Conf. Proc. 19, 1996, 681-684

[29] P. Zhang, Hochschild cohomology of truncated basic cycle, Sci. China (Ser. A), 40 (1997), 1272-1278.

[30] P. Zhang, The Hochschild homology of a truncated basic cycle, Algebra Colloq. 5 (1998), 77-84. 